\title{
Measurements of the Yield Stress in Frictionless Granular Systems
}

\author{
Ning $\mathrm{Xu}^{1}$ and Corey S. O'Hern ${ }^{1,2}$ \\ 1 Department of Mechanical Engineering, \\ Yale University, New Haven, CT 06520-8284. \\ 2 Department of Physics, Yale University, \\ New Haven, CT 06520-8120.
}

(Dated: October 4, 2018)

\begin{abstract}
We perform extensive molecular dynamics simulations of $2 \mathrm{D}$ frictionless granular materials to determine whether these systems can be characterized by a single static yield shear stress. We consider boundary-driven planar shear at constant volume and either constant shear force or constant shear velocity. Under steady flow conditions, these two ensembles give similar results for the average shear stress versus shear velocity. However, near jamming it is possible that the shear stress required to initiate shear flow can differ substantially from the shear stress required to maintain flow. We perform several measurements of the shear stress near the initiation and cessation of flow. At fixed shear velocity, we measure the average shear stress $\Sigma_{y v}$ in the limit of zero shear velocity. At fixed shear force, we measure the minimum shear stress $\Sigma_{y f}$ required to maintain steady flow at long times. We find that in finite-size systems $\Sigma_{y f}>\Sigma_{y v}$, which implies that there is a jump discontinuity in the shear velocity from zero to a finite value when these systems begin flowing at constant shear force. However, our simulations show that the difference $\Sigma_{y f}-\Sigma_{y v}$, and thus the discontinuity in the shear velocity, tend to zero in the infinite system size limit. Thus, our results indicate that in the large system limit, frictionless granular systems are characterized by a single static yield shear stress. We also monitor the short-time response of these systems to applied shear and show that the packing fraction of the system and shape of the velocity profile can strongly influence whether or not the shear stress at short times overshoots the long-time average value.

PACS numbers: $47.50 .+\mathrm{d}$ 83.10.Mj 83.50.-v 45.70.Mg,
\end{abstract}

\section{INTRODUCTION}

The static yield shear stress, or similarly the static shear modulus, is an important material property that distinguishes solids from liquids [1]. Solids possess a nonzero static yield shear stress, while it vanishes for liquids. Solids are able to resist applied shear stresses below the yield shear stress, but plastic flow occurs when shear stresses larger than the yield shear stress are applied. In contrast, liquids flow when any finite shear stress is applied.

Disordered materials such as molecular and colloidal glasses, static granular materials, and concentrated emulsions also possess a nonzero yield shear stress. However, it is difficult to determine precisely the yield shear stress in these amorphous systems since they often display nonlinear and spatially nonuniform response, for example creep flow, intermittent dynamics, and shear localization, when shear stress is applied. The value of the yield stress in these amorphous systems can also depend on how it is measured. For example, the yield shear stress required to generate steady flow in an originally unsheared system may differ significantly from a measure of the yield stress obtained by approaching the static state by slowly decreasing the shearing velocity. The yield shear stress may also depend strongly on how the system was prepared. For example, it has been shown that the yield shear stress is sensitive to the age and strain history in glassy systems [2] and the construction history [3, 4] and micro-structural details [5] in granular materials.
There have been a number of recent computational investigations of the transition from static to flowing states in granular and glassy systems. For example, measurements of the yield shear stress or static shear modulus have been conducted as a function of packing fraction in model foams [6], emulsions [7], and frictionless granular materials [8] and as a function of temperature and strain rate in dense Lennard-Jones glasses 9, 10, 11, metallic glasses [12], and polymer glasses [13, 14]. However, an important question that has not been adequately addressed by these previous studies is whether or not there is a unique measure of the static yield shear stress in amorphous granular and glassy systems. Several studies have pointed out that the shear stress required to initiate flow can be larger than the shear stress required to prevent slow shear flows from stopping [9, 15], but, does this difference in shear stress persist in the infinite system size limit? If so, what physical mechanism (for example, force chains in granular materials [16]) is responsible for the difference? If not, how significant are the finite-size effects?

We perform molecular dynamics simulations of frictionless granular materials subjected to boundary-driven shear at fixed volume to determine whether or not these simple systems can be characterized by a single static yield stress in the large system limit. At constant shearing velocity, we measure the long-time average shear stress $\Sigma_{y v}$ in the limit of zero shearing velocity. We also perform simulations at fixed shear force and identify the minimum shear stress $\Sigma_{y f}$ required to maintain steady 
shear flow at long times. We indeed find that $\Sigma_{y f}>\Sigma_{y v}$ at finite system size. However, the difference tends to zero in the infinite system size limit. Thus, we argue that large frictionless granular systems possess a single static yield shear stress. In future studies, we will include static friction to determine whether the gap $\Sigma_{y f}-\Sigma_{y v}$ remains finite in large frictional granular systems.

We also investigate the short-time response of frictionless granular systems to applied shear. Previous studies of sheared glassy systems [10, 14, 17] have found that the shear stress in response to applied shear strains overshoots the long-time average value at short times. The shear stress overshoot is often employed as a dynamic measure of the yield shear stress. In addition, these studies have found that the size of the overshoot increases with increasing shear rate and decreasing temperature. Does the shear stress overshoot at short times also occur in model granular systems? Is the shear stress overshoot related to the difference in the measured values of the yield shear stress $\Sigma_{y f}-\Sigma_{y v}$ ? To address these questions, we monitor the short-time response of the shear stress to applied shear strain over a range of packing fractions and shear velocities and in systems where we constrain the velocity profiles to be linear and in systems without such a constraint. We find that the packing fraction and shape of the velocity profile strongly influence the short time response. In fact, systems near random close packing with no constraints on the velocity profile do not possess a shear stress overshoot in the range of shear rate considered, while systems that are constrained to have linear velocity profiles do possess an overshoot.

\section{METHODS}

In this section, we provide important details of the simulation methods. We performed molecular dynamics simulations of frictionless granular systems in 2D at fixed volume in the presence of an applied shear stress. The shear stress was applied by moving a top boundary layer of particles horizontally as a rigid body at either fixed shearing velocity $u$ or fixed lateral force $F_{0}$, while the bottom boundary remained stationary. We studied systems composed of 50-50 mixtures of large and small particles with equal mass $m$ and diameter ratio 1.4. These bidisperse systems do not crystallize or segregate under shear [18, 19].

The position $\vec{r}_{i}$ of each particle $i$ in the bulk was obtained as a function of time $t$ by solving Newton's equations of motion

$$
m \frac{d^{2} \vec{r}_{i}}{d t^{2}}=\vec{F}_{i}=\sum_{j}\left[F_{i j}^{r}\left(r_{i j}\right)-b\left(\vec{v}_{i}-\vec{v}_{j}\right) \cdot \hat{r}_{i j}\right] \hat{r}_{i j},
$$

where the sum over $j$ is a sum over the nearest neighbors of particle $i$. The simple frictionless granular systems considered here interact via two pairwise forces that act only along the line connecting particle centers $\hat{r}_{i j}$ and are nonzero only when particles $i$ and $j$ overlap 20]. The first pairwise interaction is the purely repulsive linear spring force

$$
F_{i j}^{r}\left(r_{i j}\right)=\frac{\epsilon}{\sigma_{i j}}\left(1-\frac{r_{i j}}{\sigma_{i j}}\right),
$$

where $\epsilon$ is the characteristic energy scale of the interaction, $\sigma_{i j}=\left(\sigma_{i}+\sigma_{j}\right) / 2$ is the average diameter of particles $i$ and $j$, and $r_{i j}$ is their separation. The second pairwise interaction is dissipative and proportional to velocity differences along $\hat{r}_{i j}$. We chose the damping coefficient $b=0.0375$, which corresponds to a restitution coefficient $e=0.92$ typical for granular systems.

At constant velocity, the equation of motion for each particle in the top boundary is trivial, $d^{2} x / d t^{2}=0$, subject to $d x / d t=u$. At constant shear force $F_{0}$, each particle in the top boundary obeys an equation of motion similar to that in Eq. 1.

$$
M \frac{d^{2} x}{d t^{2}}=F_{0}+\sum_{i, j}\left(\vec{F}^{r}\left(r_{i j}\right)-b\left(u-\vec{v}_{j}\right) \cdot \hat{r}_{i j}\right) \hat{r}_{i j} \cdot \hat{x}
$$

where $M$ is the mass of the top boundary. The second term in Eq. [3] is the total horizontal force on particles $i$ in the top boundary arising from interactions with particles $j$ in the bulk.

The starting configurations were prepared by choosing a packing fraction $\phi=0.85$ near random close packing for this system [21] and random initial particle positions. The system was then allowed to relax at fixed volume to the nearest local energy minimum using the conjugate gradient method [22]. During the quench, periodic boundary conditions were implemented in both the $x$ and $y$-directions. Following the quench, particles with $y$ coordinates $y>L_{y}(y<0)$ were chosen to comprise the top (bottom) boundary. This preparation algorithm created rough and amorphous top and bottom boundaries, which prevents slip between the bulk and boundary particles during shear. After the boundaries are constructed, the simulation cell was nearly square and contained $N_{0}$ bulk particles and $N_{b}$ particles in the top and bottom boundaries. Periodic boundary conditions in the $x$ direction were employed during shear.

During the simulations, we calculated the shear stress on the top and bottom boundaries and in the bulk. Each of these measurements gave similar values for the average shear stress, however, the shear stress fluctuations were much larger on the boundaries as expected. Therefore, below we focus on measurements of the bulk shear stress calculated using the virial expression [23]:

$$
\Sigma=-\frac{1}{L_{x} L_{y}}\left(\sum_{i=1}^{N_{0}} \delta v_{x i} \delta v_{y i}+\frac{1}{2} \sum_{i \neq j}^{N_{0}} x_{i j} F_{y i j}\right),
$$

where $\delta \vec{v}_{i}=\vec{v}_{i}-\left\langle\vec{v}_{i}\right\rangle$ is the deviation of the velocity of bulk particle $i$ from the average velocity $\left\langle v_{i}\right\rangle$ at height $y_{i}$.

We performed several measurements of the shear stress near the initiation and cessation of flow. First, at fixed 


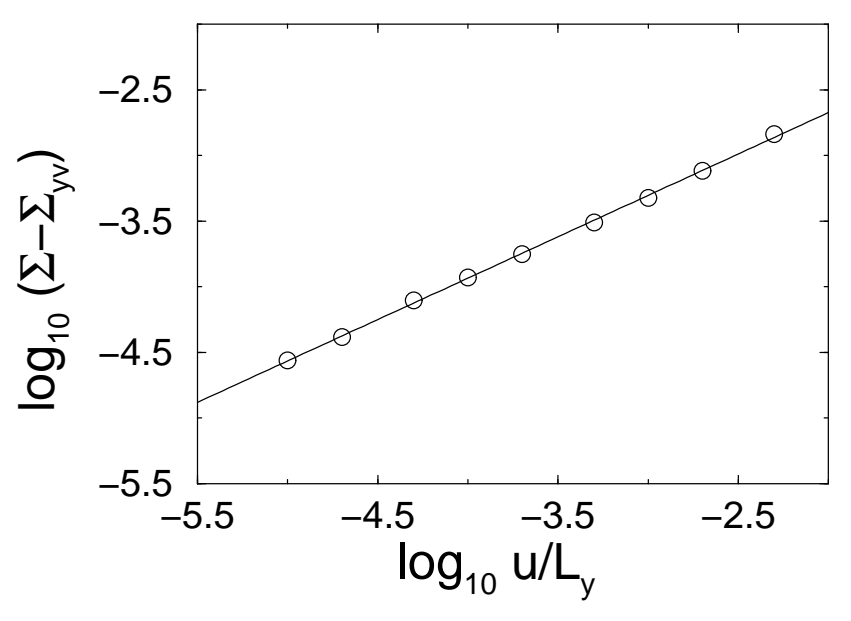

FIG. 1: Deviation in the shear stress $\Sigma$ from the shear stress $\Sigma_{y v}$ in the $u \rightarrow 0$ limit versus shear rate $u / L_{y}$ for a system with $N_{0} \approx 1024$ bulk particles sheared at constant $u$. The solid line has slope 0.63 .

shearing velocity, we measured the long-time average shear stress $\Sigma_{y v}$ in the $u \rightarrow 0$ limit. Second, at fixed lateral force, we measured the minimum shear stress $\Sigma=F_{0} / L_{x}$ required to maintain steady shear flow at long times. For all measurements of the shear stress we averaged over at least 100 different initial realizations. We did not find large differences in the shear stress response among different starting configurations. Also, to assess finite size effects, we varied the number of particles in the bulk $N_{0}$ over more than two orders of magnitude from $N_{0}=32$ to 4096 . In the subsequent discussion of results, the small particle diameter $\sigma$, characteristic energy $\epsilon$, and $\sigma \sqrt{m / \epsilon}$ were chosen as the units of length, energy, and time, and all quantities are normalized by these below.

\section{RESULTS}

In this section, we present a number of novel results from our simulations of frictionless granular materials subjected to boundary-driven planar shear.

\section{A. Constant Shearing Velocity}

We have measured the average shear stress $\Sigma$ as a function of shear rate $u / L_{y}$ in systems sheared at fixed velocity $u$ of the top boundary. At each $u$, we began with an unsheared initial configuration, the system was sheared for a strain of at least 10, and then the shear stress was averaged over an additional strain of 100 . We have shown in previous studies [19] that at such large strains these systems are spatially uniform and possess linear velocity profiles. We find that the flow curve $\left(\Sigma\right.$ versus $\left.u / L_{y}\right)$ for the system obeys the commonly used phenomenological

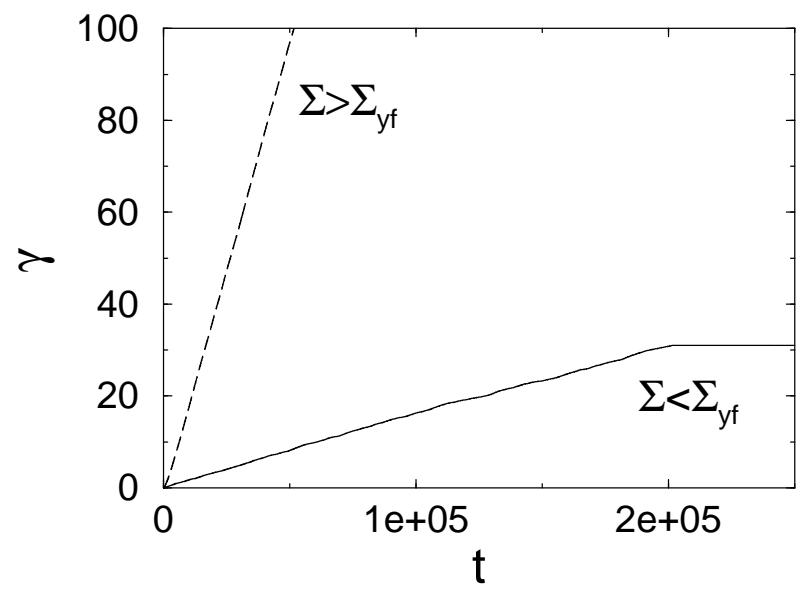

FIG. 2: Shear strain $\gamma$ versus time $t$ for a system with $N_{0} \approx$ 1024 bulk particles sheared at constant force $F_{0}=\Sigma / L_{y}$. Two shear stresses $\Sigma=3.7 \times 10^{-4}$ (solid line) and $\Sigma=9.7 \times 10^{-4}$ (dashed line) are shown. The smaller value is below and the larger is above the minimum yield stress $\Sigma_{y f}=4.4 \times 10^{-4}$ required to maintain steady flow at long times.

form [9, 10]

$$
\Sigma-\Sigma_{y v}=A_{v}\left(u / L_{y}\right)^{\alpha}
$$

where $A_{v}>0, \Sigma_{y v}$ is the shear stress in the $u \rightarrow 0$ limit, and the power-law exponent $\alpha \approx 0.63$ [24]. The flow curve for a system with $N_{0} \approx 1024$ bulk particles is shown in Fig. 11 and $\Sigma_{y v}=2.1 \times 10^{-4}$ for this system size. Systems sheared at constant velocity flow at any nonzero $u$, however, by extrapolating the flow curve to $u \rightarrow 0$, we can obtain a measure of the yield shear stress $\Sigma_{y v}$.

\section{B. Constant Shearing Force}

We also studied frictionless granular systems sheared at fixed lateral force $F_{0}$. In this ensemble, granular systems do not flow on long time scales unless the applied shear stress $\Sigma=F_{0} / L_{x}$ exceeds a shear stress threshold, $\Sigma_{y f}$. In Fig. 2 we show the shear strain $\gamma=x / L_{y}$, where $x$ is the horizontal displacement of the top boundary, as a function of time for applied shear stresses above and below $\Sigma_{y f}$ in a system with $N_{0} \approx 1024$ and averaged over 100 initial realizations. When $\Sigma>\Sigma_{y f}$, the shear strain diverges and the system flows at long times at an average shear rate that is consistent with the flow curve for the fixed shearing velocity ensemble. The average shear rate is given by the slope of strain versus time in Fig. 2. When $\Sigma<\Sigma_{y f}$, the system can flow at short time scales. However, the system is able to find a configuration that can support the applied shear stress, and the system stops flowing. Moreover, the flow will not resume because dissipation damps the velocity fluctuations. As shown in Fig. 3] the maximum shear strain $\gamma_{s}$ that the system attains increases as a power-law with the applied 


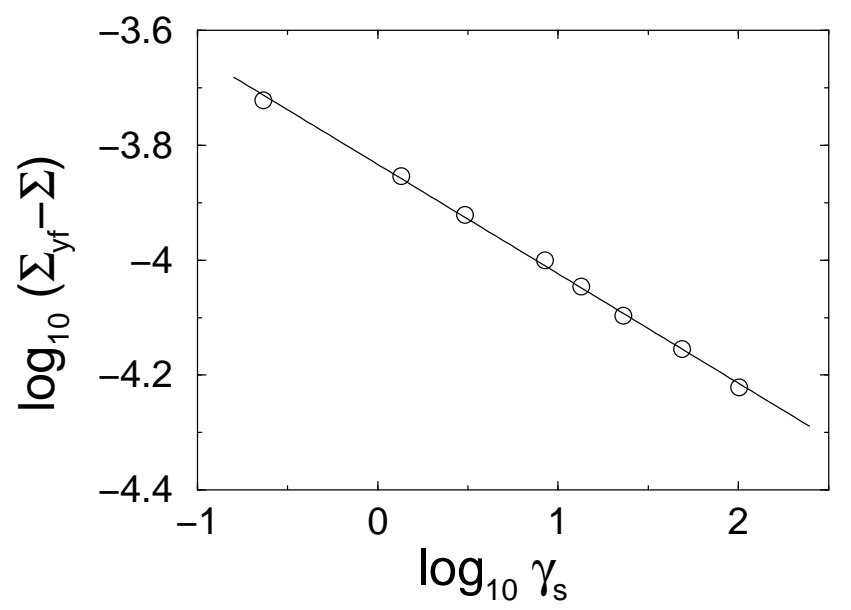

FIG. 3: The difference between the applied shear stress $\Sigma$ and the minimum shear stress $\Sigma_{y f}$ required to maintain shear flow at long times versus the maximum shear strain $\gamma_{s}$ obtained. The solid line has slope -0.20 .

shear stress $\Sigma$ and diverges as $\Sigma \rightarrow \Sigma_{y f}$ from below:

$$
\Sigma_{y f}-\Sigma=\frac{A_{f}}{\gamma_{s}^{\beta}},
$$

where $A_{f}>0, \Sigma_{y f}$ is the minimum shear stress at which $\gamma_{s} \rightarrow \infty$, and the power-law exponent $\beta \approx 0.20$. For $N_{0} \approx 1024$ bulk particles, $\Sigma_{y f}=4.4 \times 10^{-4}>\Sigma_{y v}$.

\section{System-Size Dependence}

In the previous section, we showed that $\Sigma_{y f}>\Sigma_{y v}$ for systems with $N_{0} \approx 1024$ bulk particles. How does the difference in these two measurements of the yield shear stress depend on system size? Does the difference tend to zero for frictionless granular systems? To answer these questions, we performed measurements of $\Sigma_{y v}$ and $\Sigma_{y f}$ for systems with $N_{0}$ in the range 32 to 4096. For all system sizes, the shear stress obeyed Eq. [5in the constant shear velocity ensemble and Eq. [6] in the constant shear force ensemble. We found that $\Sigma_{y f}>\Sigma_{y v}$ for all system sizes studied, however, the difference between these two measures of the yield shear stress decreased as $N_{0} \rightarrow \infty$. Both measures decreased with increasing system size and converged to the same value in the infinite system size limit, $\Sigma_{y \infty} \approx 1.7 \times 10^{-4}$. In Fig. 4 we show the systemsize dependence of $\Sigma_{y f}$ and $\Sigma_{y v}$. For example, in the constant force ensemble, $\Sigma_{y f}-\Sigma_{y \infty}$ scales as a power law with $N_{0}$

$$
\Sigma_{y f}-\Sigma_{y \infty}=\frac{B_{f}}{N_{0}^{\eta_{f}}}
$$

over the entire range of system sizes with $B_{f}>0$ and $\eta_{f} \approx 0.75 . \Sigma_{y v}$ has a similar power-law dependence for small systems, but a more rapid power-law decay with

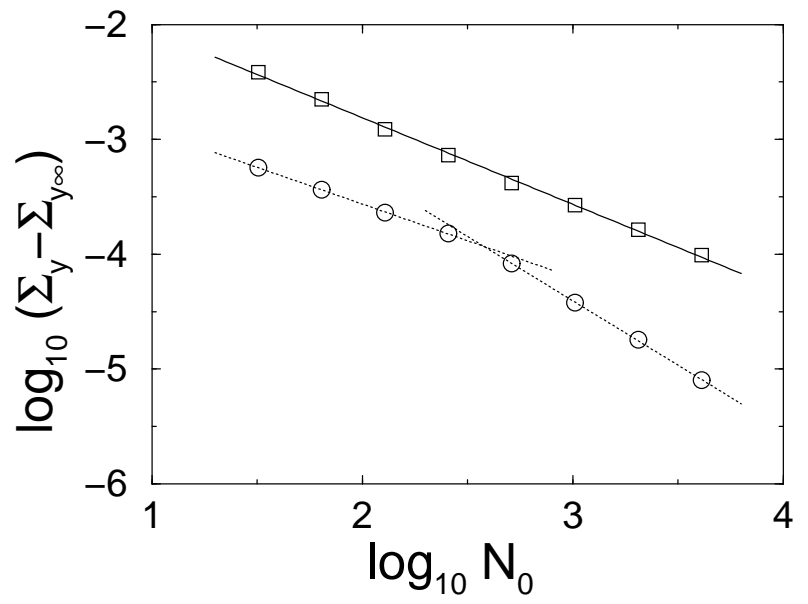

FIG. 4: The deviation of the yield shear stress $\Sigma_{y}$ from its value in the infinite system size limit $\Sigma_{y \infty}$ as a function of the number of bulk particles $N_{0}$ in the constant shear velocity (circles) and force (squares) ensembles.

$\eta_{v} \approx 1.1$ occurs for $N_{0}>300$. A simple interpretation of $\Sigma_{y f} \rightarrow \Sigma_{y v}$ is that $\Sigma_{y v}$ measures the average shear stress, while $\Sigma_{y f}$ is related to the maximum shear stress in the $u \rightarrow 0$ limit. Since the distribution of shear stresses becomes a $\delta$-function and the shear fluctuations vanish, the difference between $\Sigma_{y v}$ and $\Sigma_{y f}$ vanishes in the large system limit for frictionless granular systems.

\section{Discontinuity in the Shear Rate}

In Fig. [5 we show a comparison of the flow curves, i.e. shear stress $\Sigma$ versus shear rate $u / L_{y}$, for systems in the constant shearing velocity and force ensembles. For large shear stresses $\Sigma>\Sigma_{y f}$, there is a correspondence between shear rate and shear stress in the two ensembles. However, since $\Sigma_{y f}$ is larger than the average shear stress $\Sigma_{y v}$ in the $u \rightarrow 0$ limit at finite system size, there is a jump discontinuity in the shear rate when the applied shear stress is increased above $\Sigma_{y f}$. In fact, several recent experimental studies have found that foams, emulsions, and granular materials also display a rate of strain discontinuity when they begin flowing in response to applied shear stress [25, 26, 27]. However, we find that in frictionless granular systems, the jump discontinuity in shear rate upon initiation of shear flow is a finite size effect- $-u_{c}$ tends to zero in the infinite system size limit. The jump discontinuity $u_{c}\left(N_{0}\right)$ is obtained by solving $\Sigma_{y v}\left(u_{c}, N_{0}\right)=\Sigma_{y f}\left(N_{0}\right)$. Using the scaling relations in Eqs. [5] and 7 we find that $u_{c} \sim N_{0}^{-\eta_{f} / \alpha} \sim N_{0}^{-1.2}$, which is confirmed in the inset to Fig. In light of these results, we advocate further experimental studies of the rate of strain discontinuities in soft glassy materials, especially in planar shear cells, to determine whether there are strong finite size effects. 


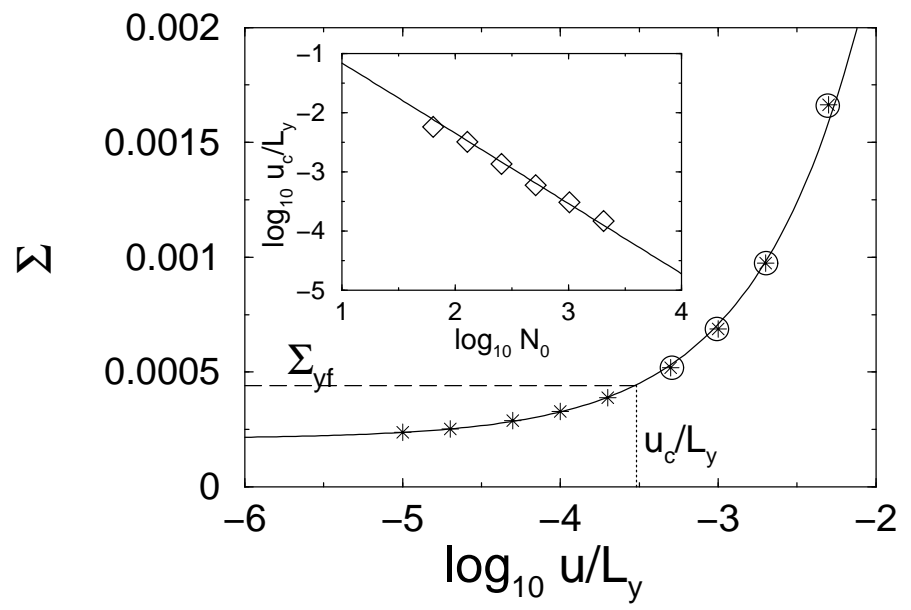

FIG. 5: Comparison of the flow curves at constant shear velocity (asterisks) and constant shear force (circles) for $N_{0} \approx$ 1024. The solid line is a fit to Eq. 5 $u_{c} / L_{y}$ is the jump discontinuity in the boundary shear rate that occurs when the system begins flowing at constant shear force. The inset shows the jump discontinuity $u_{c} / L_{y}$ as a function of $N_{0}$. The solid line has slope $\approx-1.2$.

\section{E. Shear Stress Overshoot}

A frequently used measure of the dynamic yield shear stress is the shear stress overshoot above the long-time average value in systems sheared at finite shear rate. In fact, several recent computational studies of dense Lennard-Jones glasses have measured the dependence of the shear stress overshoot on the bath temperature and imposed shear rate [10, 14, 17]. In this final section, we present results from simulations of frictionless granular systems undergoing planar shear to determine whether a significant shear stress overshoot occurs on short time scales in the slowly sheared regime in these systems.

There are several key differences between our current study and previous investigations of the shear stress overshoot in sheared glassy systems: 1) we study systems with no constraints on the velocity profile as well as systems with constraints that enforce a linear velocity profile $\left\langle v_{x}\right\rangle=u y / L_{y}$ as used in simulations of glasses [28], 2) we study a wide range of packing fractions from near random close packing at $\phi=0.85$ to overcompressed systems at $\phi=1.1$, and 3) we focus on dissipative, granular systems, not conservative, thermal systems. In the results below, we show that the packing fraction and shape of the velocity profile strongly influence the short-time response of sheared granular systems. We fixed the dissipation in this study, however, the influence of dissipation will be investigated in a future study [29].

To study the shear stress overshoot, we measured the shear stress response of the system at small strains to a slow applied shear rate $u / L_{y}=10^{-4}$. The shear stress as a function of shear strain averaged over 100 independent realizations is shown in Fig. [6] for a system with $N_{0} \approx$
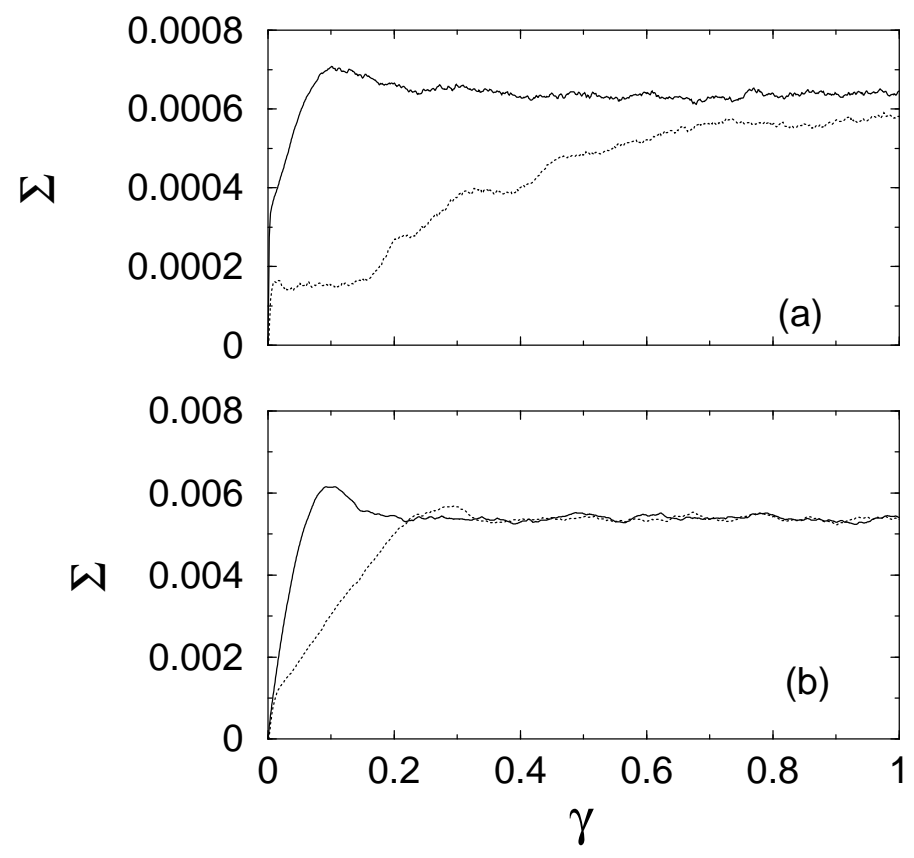

FIG. 6: Shear stress $\Sigma$ versus shear strain $\gamma$ for systems with $N_{0} \approx 4096$ at (a) $\phi=0.85$ and (b) $\phi=1.10$ and $u / L_{y}=$ 0.001 . The solid and dotted lines show results for systems with and without a constraint that enforces linear velocity profiles.

4096. Note that in Fig. [6] the shear stress is measured only over small shear strains up to $\gamma=1$. In contrast, the shear stress was averaged over large shear strains up to $\gamma=100$ in previous figures.

Two striking results are presented in Fig. 6 First, when the velocity profile is unconstrained, the shear stress does not overshoot the long-time average shear stress at this shear rate. The shear stress increases monotonically to the long-time average value. The shear strain required to reach the long-time average shear stress decreases with increasing $\phi$, but is much less than the shear strain required for the system to possess a linear velocity profile. See Ref. [19] for an extensive discussion of the evolution of the velocity profiles in sheared granular systems. In contrast, when we constrain the system to possess a linear velocity profile, a large shear stress overshoot develops. In addition, the shear strain required to reach the long-time average shear stress is $<0.2$ and roughly independent of packing fraction. These results suggest that the shear stress overshoot at short times in glassy and granular systems is an artifact of the fact that the velocity field is constrained to be linear. At the very least, the constraint significantly amplifies and speeds up the response of the system to applied shear. Second, the packing fraction strongly influences the height of the shear stress overshoot. The overshoot for $\phi=0.85$ in Fig. [6 (a) (with the constraint) is nearly a factor of 10 smaller than that for $\phi=1.1$ in Fig. 6 (b). Moreover, if we define $\Delta=\left(\Sigma_{m}-\Sigma_{p}\right) / \Sigma_{p}$ as the relative 
height of the shear stress overshoot with maximum shear stress $\Sigma_{m}$ and shear stress plateau at long times $\Sigma_{p}$, $\Delta \approx 0.09$ at $\phi=0.85$ compared to $\Delta \approx 0.15$ at $\phi=1.10$. Both of these findings demonstrate that the shear stress overshoot is less pronounced in frictionless granular systems near random close packing with the local dissipation model in Eq. 1 than in dense Lennard-Jones glasses.

\section{CONCLUSION}

In this article, we studied model frictionless granular systems near the initiation and cessation of shear flow using molecular dynamics simulations of boundary-driven shear flow at constant volume in $2 \mathrm{D}$. These simulations were performed to address several open questions concerning the jamming (or unjamming) transition in frictionless granular systems. First, we wanted to determine whether these model systems can be characterized by a single yield shear stress in the large system limit. We compared two measures of yield shear stress: 1) the average shear stress $\Sigma_{y v}$ in the limit where the velocity of the shearing boundary tends to zero and 2) the minimum shear stress $\Sigma_{y f}$ required for steady shear flow at long times when a constant force is applied to the shearing boundary. As found in previous studies of glassy and granular systems, $\Sigma_{y f}>\Sigma_{y v}$ in finite-sized systems. However, these two measures become identical $\Sigma_{y f}=\Sigma_{y v}$ in the infinite system size limit in frictionless granular systems. An important direction for future research is to determine how the inclusion of static frictional forces affects these results. Does the difference $\Sigma_{y f}-\Sigma_{y v}$ remain finite in the large system limit in frictional granular systems? Recent studies have argued that a large yield stress difference $\Sigma_{y f}-\Sigma_{y v}$ is responsible for shear banding - spatially localized velocity profilesin dense Lennard-Jones glasses [15]. However, our results suggest that another mechanism is responsible for shear banding in frictionless granular systems [18, 19]. Further studies are required to determine whether a possible gap $\Sigma_{y f}-\Sigma_{y v}>0$ contributes to shear banding in large frictional systems.

Another question addressed in this article is whether frictionless granular systems possess a strain rate discontinuity when they begin flowing at constant force as has been found in several recent experimental studies on similar systems [25, 26, 27]. We indeed found a discontinuity in the shear rate upon the initiation of flow, but the discontinuity is proportional to $\Sigma_{y f}-\Sigma_{y v}$ and therefore tends to zero in the infinite system size limit. We recommend further experimental studies in planar shear cells to assess the finite size effects on the strain rate discontinuity.

We have also investigated the short-time response of the shear stress of the system when the shearing boundary is driven at fixed velocity. It is well-known for glassy systems that the shear stress at short times can overshoot the long-time average value when these systems are sheared at finite shear rate, and the height of the overshoot is often used as a measure of the dynamic yield shear stress. We found several novel results for the shorttime response. First, the shape of the velocity profile strongly influences the shear stress at short times. When our systems did not have a constraint imposed on the velocity profile, we did not observe an overshoot in the shear stress. In contrast, when a linear velocity profile was enforced, a strong shear stress overshoot occurred. Second, the height of the overshoot (at least in systems with linear velocity profiles) decreases with decreasing packing fraction. For example, the height of the peak in shear stress is a factor of 10 smaller near random close than in an overcompressed system at $\phi=1.1$. These results point out that there is not a significant shear stress overshoot in frictionless granular systems - in contrast to dense Lennard-Jones glasses.

Acknowledgments Financial support from NSF grant numbers CTS-0456703 (CSO) and DMR-0448838 (NX,CSO) is gratefully acknowledged. We also thank Yale's High Performance Computing Center for generous amounts of computer time.
[1] Jamming and Rheology ed. A. J. Liu and S. R. Nagel (Taylor \& Francis, N. Y., 2001).

[2] M. Utz, P. G. Debenedetti, and F. H. Stillinger, Phys. Rev. Lett. 84, 1471 (2000).

[3] E. R. Nowak, J. B. Knight, E. Ben-Naim, H. M. Jaeger, and S. R. Nagel, Phys. Rev. E 57, 1971 (1998).

[4] L. Vanel, D. Howell, D. Clark, R. P. Behringer, and E. Clement, Phys. Rev. E 60, R5040 (1999).

[5] W. Losert, J. C. Geminard, S. Nasuno, and J. P. Gollub, Phys. Rev. E 61, 4060 (2000).

[6] D. J. Durian, Phys. Rev. Lett. 75, 4780 (1995); Phys. Rev. E 55, 1739 (1997).

[7] T. G. Mason, M.-D. Lacasse, G. S. Grest, D. Levine, J. Bibette, and D. A. Weitz, Phys. Rev. E 56, 3150 (1997).
[8] C. S. O'Hern, S. A. Langer, A. J. Liu, and S. R. Nagel, Phys. Rev. Lett. 88, 075507 (2002); C. S. O'Hern, L. E. Silbert, A. J. Liu, and S. R. Nagel, Phys. Rev. E 68, 011306 (2003).

[9] F. Varnik, L. Bocquet, and J.-L. Barrat, J. Chem. Phys. 120, 2788 (2004).

[10] J. Rottler and M. O. Robbins, Phys. Rev. E 68, 011507 (2003).

[11] J. Rottler and M. O. Robbins, Phys. Rev. Lett. 95, 225504 (2005).

[12] X. Fu, D. A. Rigney, and M. L. Falk, J. Non-Cryst. Solids 317, 206 (2003).

[13] G. He and M. O. Robbins, Tribol. Lett. 10, 7 (2001); Phys. Rev. B 64, 035413 (2001). 
[14] J. Rottler and M. O. Robbins, Phys. Rev. E 64, 051801 (2001).

[15] F. Varnik, L. Bocquet, J.-L. Barrat, and L. Berthier, Phys. Rev. Lett. 90, 095702 (2003); L. Berthier and J.-L. Barrat, J. Chem. Phys. 116, 6228 (2002).

[16] T. S. Majmudar and R. P. Behringer, Nature 435, 1079 (2005).

[17] F. Albano, N. Lacevic, M. L. Falk, and S. C. Glotzer, Materials Science and Engineering A 375, 671 (2004).

[18] N. Xu, C. S. O'Hern, and L. Kondic, Phys. Rev. Lett. 94, 016001 (2005).

[19] N. Xu, C. S. O'Hern, and L. Kondic, Phys. Rev. E. 72, 041504 (2005).

[20] S. Luding, Phys. Rev. E 55, 4720 (1994).

[21] N. Xu, J. Blawzdziewicz, and C. S. O'Hern, Phys. Rev. E 71, 061306 (2005).

[22] W. H. Press, B. P. Flannery, S. A. Teukolsky, and W. T. Vetterling, Numerical Recipes in Fortran 77 (Cambridge
University Press, New York, 1986).

[23] M. P. Allen and D. J. Tildesley, Computer Simulations of Liquids, (Oxford University Press, Oxford, 1987).

[24] The flow exponent $\alpha$ depends on the packing fraction $\phi$. For dilute Newtonian systems, $\alpha=1$ and decreases with increasing $\phi$.

[25] F. Da Cruz, F. Chevoir, D. Bonn, and P. Coussot, Phys. Rev. E 66, 051305 (2002).

[26] J. Lauridsen, G. Chanan, and M. Dennin, Phys. Rev. Lett. 93, 018303 (2004).

[27] M. Dennin, Colloids and Surfaces A 263, 76 (2005).

[28] See a discussion of the Sllod equations of motion employed in simulations of sheared liquids and glasses, for example, in D. J. Evans and G. P. Morriss, Statistical Mechanics of Nonequilibrium Liquids, (Academic Press, London, 1990).

[29] N. Xu and C. S. O'Hern, unpublished. 